\title{
A stabilized Kuramoto-Sivashinsky system
}

\author{
Boris A. Malomed \\ Department of Interdisciplinary Studies, Faculty of Engineering, \\ Tel Aviv University, Tel Aviv 69978, Israel \\ Bao-Feng Feng \\ Department of Mathematics, the University of Kansas, \\ Lawrence, KS 66045, U.S.A. \\ Takuji Kawahara \\ Department of Aeronautics and Astronautics, \\ Graduate School of Engineering, \\ Kyoto University, Sakyo-ku, Kyoto 606-8501, Japan
}

October 31, 2018

\begin{abstract}
A model consisting of a mixed Kuramoto - Sivashinsky - Korteweg - de Vries equation, linearly coupled to an extra linear dissipative equation, is proposed. The model applies to the description of surface waves on multilayered liquid films. The extra equation makes its possible to stabilize the zero solution in the model, thus opening way to the existence of stable solitary pulses (SPs). By means of the perturbation theory, treating dissipation and instability-generating gain in the model (but not the linear coupling between the two waves) as small perturbations, and making use of the balance equation for the net momentum, we demonstrate that the perturbations may select two steady-state solitons from their continuous family existing in the absence of the dissipation and gain. In this case, the selected pulse with the larger value of the amplitude is expected to be stable, provided that the zero solution is stable. The prediction is completely confirmed by direct simulations. If the integration domain is not very large, some pulses are stable even when the zero background is unstable. An explanation to the latter finding is proposed. Furthermore, stable bound states of two and three pulses are found numerically.
\end{abstract}

PACS: $05.45 . \mathrm{Yv}, 46.15 . \mathrm{Ff}$ 


\section{Introduction}

The Kuramoto - Sivashinsky (KS) equation is a well-known model of onedimensional turbulence, which was derived in various physical contexts, including chemical-reaction waves [1], propagation of combustion fronts in gases [2], surface waves in a film of a viscous liquid flowing along an inclined plane [3], patterns in thermal convection 沟, rapid solidification [5], and others. It has the form

$$
u_{t}+u u_{x}=-\alpha u_{x x}-\gamma u_{x x x x}
$$

where $\alpha>0$ and $\gamma>0$ are coefficients accounting for the long-wave instability (gain) and short-wave dissipation, respectively.

A generalized form of the KS equation contains a linear dispersive term borrowed from the Korteweg - de Vries (KdV) equation,

$$
u_{t}+u u_{x}+u_{x x x}=-\alpha u_{x x}-\gamma u_{x x x x} .
$$

As well as the KS equation proper, the generalized equation (2) applies to the description of surface waves on flowing liquid films [6], and it also serves as a general model which allows one to study various nonlinear dissipative waves [7]- 10]. In particular, a subject of considerable interest was the study of solitarypulse (SP) solutions to both the KS equation [11] and Eq. (2) [8, 10. By means of numerical methods, it is possible to find a vast family of SP solutions to Eqs. (11) and (2) in the form $u(x, t)=u(x-s t)$ with a constant velocity $s$. However, it is obvious that all these solutions are unstable in an infinitely long system, as the zero solution, into which SP goes over at $|x-s t| \rightarrow \infty$, is unstable in both equations.

It is an issue of a principal interest to find a physically relevant model which combines the dissipative and dispersive features, and simultaneously supports stable SPs. It appears that the simplest possibility to construct such a model is to couple Eq. (2) to an extra linear stabilizing equation, arriving at a system

$$
\begin{aligned}
u_{t}+u u_{x}+u_{x x x} & =-\alpha u_{x x}-\gamma u_{x x x x}+\epsilon_{1} v_{x}, \\
v_{t}+c v_{x} & =\Gamma v_{x x}+\epsilon_{2} u_{x},
\end{aligned}
$$

where the dissipative parameter (effective diffusion coefficient) $\Gamma>0$ accounts for the stabilization (see below), and $c$ is a group-velocity mismatch between the two wave modes. The coupling parameters $\epsilon_{1}$ and $\epsilon_{2}$ must have the same sign (otherwise the coupling gives rise to an instability), while their magnitudes may be different. However, it is always possible to make them equal, $\epsilon_{1}=\epsilon_{2} \equiv \epsilon$, by means of an obvious rescaling of $u$ and $v$. Then, using the remaining scaling invariance of the equations, it is possible to set $\epsilon \equiv 1$. Thus, we will be dealing with a system containing four irreducible parameters,

$$
\begin{aligned}
u_{t}+u u_{x}+u_{x x x}-v_{x} & =-\alpha u_{x x}-\gamma u_{x x x x} \\
v_{t}+c v_{x}-u_{x} & =\Gamma v_{x x} .
\end{aligned}
$$


Note that the system conserves two "masses",

$$
M=\int_{-\infty}^{+\infty} u(x) d x, N=\int_{-\infty}^{+\infty} v(x) d x
$$

The system of equations (3) and (4) can find their natural physical realization as a model describing coupled surface and interface waves in a two-layered flowing liquid film, cf. the similar interpretation of the single equation (11) or Eq. (2) mentioned above. In particular, the linear coupling via the first derivatives is the same as in known models of coupled internal waves propagating in multilayered fluids [12]. Then, the linear dissipative equation (4) implies that the substrate layer is essentially more viscous than the upper one. In fact, the additional equation (6) may also be nonlinear, but it can be checked that inclusion of the nonlinear term $v v_{x}$ into this equation does not produce any conspicuous difference, therefore we focus on the simplest model (5), (6), which provides for the stabilization of SPs.

The system of equations (5) and (6) is qualitatively similar to a system of linearly coupled Ginzburg-Landau (GL) equations describing propagation of localized pulses in an fiber-optic core equipped with distributed gain, which is linearly coupled to an extra lossy core that provides for the stability of the pulses [13, 14, 15] (such double-core systems have recently become available to experimental studies, and they have very promising features for applications to optical communications, see a short overview in Ref. 16]). The most fundamental version of this GL system is that in which the extra stabilizing equation is also linear, cf. Eq. (6); in that case, SP solutions can be found in an exact analytical form, and they are stable in a certain parametric region [14].

In this work, we will find stable SPs in the system (5), (6), which appears to be the first example of stable pulses in a model of the KS type. In section 2 , we analyze the stability of the zero solution, which, as it was mentioned above, is a necessary condition for the stability of SPs in the infinitely long system. In section 3, an analytical perturbation theory for the pulses is developed, which is based on treating the gain and dissipation constants $\alpha, \gamma$, and $\Gamma$ in Eqs. (5) and (6) as small parameters (while the group-velocity mismatch $c$ need not be small). In the zero-order approximation, $\alpha=\gamma=\Gamma=0$, Eqs. (5) and (6) have a one-parameter family of exact soliton solutions. Using the known approach based on the balance equation for the momentum [7], we demonstrate that the combination of the perturbation terms in Eqs. (5) and (6) may select one or two stationary pulses out of the continuous family existing in the zero-order approximation. As is known [13], the existence of two different SP solutions is a necessary condition for the stability of one of them, the second pulse (the one with smaller amplitude) being unstable, as it plays the role of a separatrix between attraction domain of the zero solution and stable pulse.

In section 4 , we present results of direct numerical simulations of the full system (5), (6), which demonstrate that stable SPs exist indeed. In fact, simulations sometimes produce stable pulses even in the case when the zero solution is not stable. This stability extension may be explained by the finite size of 
the simulation domain. Moreover, stable bound states (BSs) of two and three pulses are found numerically too.

\section{The stability of the zero solution}

As it was explained above, it is necessary to investigate the stability of the trivial solution, $u=v=0$, before the consideration of pulses. To this end, we substitute into the linearized equations (5) and (6) a perturbation in the form $u \sim \exp (i k x+\lambda t), v \sim \exp (i k x+\lambda t)$, where $k$ is an arbitrary real wave number of the perturbation, and $\lambda$ is the corresponding instability growth rate, which leads to a dispersion equation,

$$
\left(\lambda-i k^{3}-\alpha k^{2}+\gamma k^{4}\right)\left(\lambda+i c k+\Gamma k^{2}\right)+k^{2}=0 .
$$

The stability condition states that both solutions of the quadratic equation (8) must satisfy the inequality $\operatorname{Re}[\lambda(k)] \leq 0$ at all the real values of $k$.

For $k \rightarrow 0$, solutions to Eq. (8) can be found in the form of an expansion

$$
\lambda(k)=i \lambda_{1} k+\lambda_{2} k^{2}+\ldots,
$$

where $\lambda_{1}=\left(-c \pm \sqrt{c^{2}+4}\right) / 2$, and

$$
\lambda_{2}=\frac{-(\Gamma-\alpha) \sqrt{c^{2}+4} \pm(\Gamma+\alpha) c}{2 \sqrt{c^{2}+4}},
$$

the sign \pm being the same in $\lambda_{1}$ and $\lambda_{2}$. As $\lambda_{1}$ is always real, at the first order the expansion (9) implies neutral stability. The expression (10) yields a necessary condition for the stability of the zero solution at the second order of the expansion, $\operatorname{Re} \lambda_{2} \leq 0$, which can be cast into a form

$$
\Gamma-\alpha \geq \sqrt{\alpha \Gamma}|c| .
$$

In the particular case $c=0$, the condition (11) amounts to $\Gamma>\alpha$, which has a simple meaning: the stabilizing diffusion coefficient in Eq. (6) must be larger than the instability-driving "anti-diffusion" (gain) coefficient in Eq. (5). A very similar necessary stability condition is known in the above-mentioned system of coupled GL equation describing a dual-core optical fiber with one active and one passive cores [13, 14].

Comprehensive analysis of the zero-solution stability was performed by means of numerical solution of the dispersion equation (8). It was found that the full stability condition does not amount to the inequality (11) (i.e., it may happen that $\operatorname{Re}[\lambda(k)]$ is negative at small $k$, but it takes positive values in some interval of finite values of $k$ ). Numerically found stability borders in the plane of the parameters $(\alpha, \Gamma)$ for a fixed value $\gamma=0.05$ of the short-wave stabilization parameter in Eq. (5) and two different values of the group-velocity mismatch, $c=0$ and $c=-1$, are shown by dashed curves in stability diagrams for the pulses displayed in Figs. 1 and 2. In both cases, the zero solution is stable to the left of the stability border. Note that, for small $\alpha$ and $\Gamma$, the zero-solution stability region is indeed determined by Eq. (11), but at larger values of $\alpha$ and $\Gamma$ there appear additional stability restrictions. 


\section{The perturbation theory for solitary pulses}

At the zeroth order, setting $\gamma=\Gamma=\alpha=0$ in Eqs. (5) and (6), but keeping an arbitrary value of $c$, we arrive at a conservative system consisting of the KdV equation coupled to an extra linear one,

$$
u_{t}+u u_{x}+u_{x x x}=v_{x}, v_{t}+c v_{x}=u_{x} .
$$

Equations (12) have a family of exact two-component soliton solutions,

$$
u(x, t)=12 \eta^{2} \operatorname{sech}^{2}(\eta(x-s t)), v(x, t)=(c-s)^{-1} \cdot u(x, t),
$$

where $\eta$ is an arbitrary parameter which determines the soliton's amplitude and width, and the velocity $s$ takes two different values for given $\eta$,

$$
s=\frac{1}{2}\left[\left(c+4 \eta^{2}\right) \mp \sqrt{\left(c-4 \eta^{2}\right)^{2}+4}\right] .
$$

It will be more convenient to use, as a parameter of the soliton family, not the amplitude $\eta$, but rather the relative velocity,

$$
\delta \equiv c-s,
$$

in terms of which the amplitude is given by an expression obtained from Eq. (14),

$$
4 \eta^{2}=c-\delta+1 / \delta
$$

The range of meaningful values of $\delta$ is restricted by the condition $\eta^{2}>0$.

We have checked by direct simulations of Eqs. (12) that all the soliton solutions (13) are stable within the framework of the unperturbed equations (12). On the other hand, simulations also clearly demonstrate that collisions between solitons having different velocities are inelastic (although not strongly inelastic, see a typical example in Fig. 3), hence the conservative system (12), unlike the $\mathrm{KdV}$ equation proper, is not an exactly integrable one. The nonintegrability of the system (12) has also been confirmed by analysis of its symmetries performed by G. Burde (unpublished).

The next step is to restore the small dissipative and gain perturbations, getting back from Eqs. (12) to Eqs. (5) and (6). To this end, we notice that the unperturbed equations (12) conserve not only the masses (7) but also the net momentum,

$$
P=\frac{1}{2} \int_{-\infty}^{+\infty}\left(u^{2}+v^{2}\right) d x .
$$

Following the work [7], in the first approximation of the perturbation theory the evolution of the soliton may be described by means of the balance equation for the momentum. Indeed, a consequence of Eqs. (5) and (6) is the following exact evolution equation for the net momentum in the presence of the perturbations:

$$
\frac{d P}{d t}=\int_{-\infty}^{+\infty}\left(\alpha u_{x}^{2}-\gamma u_{x x}^{2}-\Gamma v_{x}^{2}\right) d x
$$


Steady-state SPs are selected by the condition $d P / d t=0$. The right-hand side of Eq. (18) can be explicitly calculated in the approximation in which $u$ and $v$ are substituted by the expressions (13). After a straightforward algebra, the equation $d P / d t=0$ can be cast into the form of a cubic equation for the relative velocity $\delta$ of the unperturbed soliton (13),

$$
5 \delta^{3}+(7 \widetilde{\alpha}-5 c) \delta^{2}-5 \delta-7 \widetilde{\Gamma}=0, \widetilde{\alpha} \equiv \alpha / \gamma, \widetilde{\Gamma} \equiv \Gamma / \gamma .
$$

Roots of Eq. (19) select SPs that may exist as steady states within the framework of the perturbation theory. Note that, besides the obvious condition that physical roots for $\delta$ must be real (they may be both positive and negative), they must also satisfy a condition that, after the substitution into Eq. (16), they must produce $\eta^{2}>0$. Generally speaking, there may exist up to three physical roots of Eq. (19); however, in a vast parametric area considered, we have never encountered a case when Eq. (19) would indeed have three physical roots, while the existence of two physical solutions is quite possible, see below. As it was mentioned in the Introduction, one may expect that SP may be stable if precisely two different pulses exist, then the one with the larger amplitude $\eta^{2}$ has a chance to be stable, while the pulse with the smaller amplitude is always unstable 13, 14. Indeed, if there is a stable SP, we are dealing with a bistable system, as the parameters are chosen so that the zero solution is also stable, see the previous section. In a bistable system, there should exist a separatrix, i.e., a border between attraction domains of two stable solutions, the separatrix itself being an unstable stationary solution. In the situation with two different stationary SP solutions predicted by the perturbation theory, the one with the smaller amplitude and larger width is a natural candidate to the role of the unstable separatrix solution, while its counterpart with the larger amplitude and smaller width may be stable (generally speaking, it may be stable in a part of the parametric region where this situation takes place [13, 14]).

Equation (19) may be simplified in the case $c=0$, if we additionally assume that both renormalized parameters $\widetilde{\alpha}$ and $\widetilde{\Gamma}$ are large (in fact, we are interested in the case when $\widetilde{\Gamma} \sim \widetilde{\alpha}^{3} \gg 1$ ). Then, the term $-5 \delta$ may be neglected in Eq. (19), so that it takes the form

$$
5 \delta^{3}+7 \widetilde{\alpha} \delta^{2}-7 \widetilde{\Gamma}=0 .
$$

In the case $c=0$, the condition $\eta^{2}>0$ following from Eq. (16) also takes a simple form: a physical root is that which belongs to either of the two intervals,

$$
\delta<-1 ; 0<\delta<1 .
$$

With regard to the assumption that $\widetilde{\Gamma}$ and $\widetilde{\alpha}$ are large, it is easy to see that the simplified equation (20) always has a real root in the region $\delta>1$, which is unphysical according to Eq. (21). Two physical roots $\delta<-1$ exist under the condition

$$
\widetilde{\Gamma}<\frac{1}{3}\left(\frac{14}{15}\right)^{2} \widetilde{\alpha}^{3} .
$$


Note that this condition does not contradict the necessary condition (11) of the stability of the zero solution, which in the present case $(c=0)$ takes the form $\widetilde{\Gamma}>\widetilde{\alpha}$. Indeed, the latter inequality is compatible with (22), provided that $\widetilde{\alpha}>\sqrt{3}(15 / 14) \approx 1.856$, which is correct, as we are here dealing with the case when $\widetilde{\alpha}$ is large. However, the inequality $(22)$ is not necessarily compatible with the full stability condition for the zero solution, see Fig. 1.

In the general case, it is easy to solve Eq. (19) numerically. Then, selecting a parametric region in which there are exactly two physical solutions (which, as it was explained above, is a necessary condition for the existence of a stable SP), one may identify a narrower region in which this condition holds and, simultaneously, the zero solution is stable. Stable pulses may exist only inside that region where both necessary stability conditions overlap, and direct simulations show that all the pulses belonging to the region are stable indeed, at least in case displayed in Fig. 1, see details below.

The so defined regions in the parametric plane $(\alpha, \Gamma)$, in which stable SPs are expected, are displayed, for $\gamma=0.05$, in Figs. 1 and 2 for $c=0$ and $c=-1$, respectively. The condition of the existence of exactly two different physical solutions for the pulses holds to the right of the continuous curve in these figures [note that the part of the curve corresponding to sufficiently large values of $\Gamma$ is well approximated by the analytical expression (22) obtained above]. At the points belonging to the continuous curve, the two physical solutions merge and disappear via a typical tangent (saddle-node) bifurcation.

The same analysis performed for values of the short-wavelength-dissipation parameter $\gamma$ different from the value 0.05 , for which the results are presented in Figs. 1 and 2, shows that variation of $\gamma$ produces little change in terms of the expected SP stability region (generally, the size of the region increases with $\gamma$ ). As for the effect of the group-velocity mismatch $c$, we have found that the stability region quickly shrinks with the increase of $c$ when $c$ is positive, and there is no stability region at $c>c_{\mathrm{cr}}$, where $c_{\mathrm{cr}}$ is slightly larger than 0.3 . In that case, the areas in which, respectively, the zero solution is stable, and there are two different stationary SPs, do not overlap. To illustrate this point, a very narrow stability region in the $(\alpha, \Gamma)$ plane for $c=0.3$ is shown in Fig. 4.

\section{Numerical simulations of the solitary pulses}

As it was said above, it is necessary to directly check whether stable SPs indeed exist in the region where the stability is expected. To this end, Eqs. (5), (6) with periodic boundary conditions (b.c.) were integrated by means of an implicit Fourier spectral method [19], the time step being, typically, 0.01 and 0.02 . (a description of the method is given in the appendix). The initial conditions were taken as suggested by the perturbation theory, i.e., in the form (13), but with arbitrary values of the amplitude, in order to check whether strongly perturbed pulses relax to stable ones, i.e., whether the stable pulses are attractors.

Results are displayed in Fig. 1 by means of the symbols $\mathbf{x}$ and $\mathbf{o}$, standing for unstable and stable solitons, respectively (it may happen that, near the border 
with the unstable SPs, some pulses which appear to be stable are subject to a very weak instability which does not manifest itself within the integration time limits). As is seen, the pulses are indeed stable everywhere inside the expected stability region. Moreover, all the stable pulses were found to be strong attractors. For instance, in the case $\alpha=0.1, \Gamma=0.15$, the initial pulses definitely relaxed to a single stationary SP if their initial amplitude $A_{0}$ exceeded 1.7. In particular, starting with $A_{0}=3$ and $A_{0}=12$ at $t=0$, the pulse attained the value of the amplitude, respectively, $A=6.91$ and $A=7.18$ at $t=400$. The analytical prediction for the amplitude of the steady-state pulse (13) (the one with the larger value of the amplitude) is, at the same values of the parameters, $A_{\text {anal }} \equiv 12 \eta^{2} \approx 6.45$, so that a discrepancy with the numerical results is less than $10 \%$. On the other hand, if the initial amplitude was too small, e.g., $A_{0}=0.75$, the pulse decayed into zero, which is natural too, as the zero solution has its own attraction basin. Note that for the second (smaller) steady-state pulse, which is expected to play the role of the separatrix between the attraction basins of the stable pulse and zero solution, the perturbation theory predicts, in the same case, the amplitude $\widetilde{A}_{\text {anal }} \approx 2.15$, so it seems quite natural that the initial pulses with $A_{0}=3$ and $A_{0}=0.75$ relax, respectively, to the stable pulse and to zero.

Figure 1 shows that the numerically found upper border of the stablepulse region is quite close to the border of the existence region for the steadystate pulses, as predicted by the perturbation theory. Unlike this, the numerically identified stability region extends far below the analytically found border of the zero-solution instability. For instance, it was found that, at $\alpha=0.15$ and $\Gamma=0.2$, when the zero solution is unstable against perturbations with finite wavenumbers $k$, the fastest growing perturbation corresponding to $k=k_{\max } \approx 1.3$, a fairly stable pulse with the amplitude $A=11.75$ was found in the simulations, the pulse's amplitude predicted by the perturbation theory being $A_{\text {anal }} \approx 11.61$ in this case. Moreover, we ran simulations in which the most dangerous perturbation with the above-mentioned wavenumber $k=1.3$ and a rather large amplitude, $A_{\text {pert }}=1$, was deliberately added to the pulse in the initial configuration. Instead of growing and destroying the pulse, the perturbation was suppressed by the pulse, which remained stable indefinitely long (Fig. 5).

A cause of this extended stability can be understood. A similar feature was reported, for a generalized asymmetric (with respect to the reflection $x \rightarrow-x$ ) cubic-quintic GL equation with periodic b.c., which has moving-pulse solutions, in Ref. [17]. An explanation was that the moving pulse, traveling across the integration domain, periodically passes each point and suppresses the perturbation at a rate which exceeds the perturbation growth rate (see also Ref. [10], where stable pulses were observed in the KS - KdV equation; in that work, an explanation was that the moving pulse was able to escape growing perturbation wave packets). It seems very plausible that a similar "sweeping" mechanism explains the anomalous pulse stability in the present model. Indeed, when we repeated the simulations for the same values of the parameters but in a spatial domain four times as large (i.e., the corresponding sweeping rate is four times as small), the pulse demonstrated the expected instability, even without any 
specially added perturbation seed, see Fig. 6 .

Similar to a result reported for a system of linearly coupled Ginzburg-Landau (GL) equations in Ref. [18], bound states (BSs) with two or more peaks can be found in the present model, in addition to single SPs. To this end, we took an initial configuration constructed as a set of two or more identical exact solutions of the unperturbed system (12) with a certain separation between them. The simulations have shown that BSs featuring two or more peaks of equal amplitudes indeed develop and propagate stably. These results for the two-peak and three-peak BSs are illustrated by Figs. 7 and 8, respectively. We have also checked that, even if the amplitudes of the initial pulses and separations between them are changed, the same BS consisting of equidistant equal-amplitude peaks finally develops, i.e., the BSs are fairly robust dynamical objects. In this connection, it is relevant to mention that, in the above-mentioned coupled GL equations, only two-pulse BSs are completely stable, while BSs of three pulses are split by perturbations breaking their symmetry 18 .

\section{Conclusion}

In this work, we have introduced a model based on the Kuramoto - Sivashinsky - Korteweg - de Vries equation, which is linearly coupled to an extra linear dissipative equation. The model can be applied to the description of coupled surface and interface waves on flowing multilayered liquid films. The additional linear equation makes its possible to stabilize the zero solution, which opens way to the existence of stable solitary pulses. Treating the dissipation and gain in the model (but not the linear coupling between the two wave modes) as small perturbations, and making use of the balance equation for the net momentum, we have found that the condition of the balance between the gain and dissipation may select two steady-state solitons from their continuous family existing in the absence of the dissipation and gain (the family was found in an exact analytical form). When the zero solution is stable and, simultaneously, two SPs are picked up by the balance equation for the momentum, the pulse with the larger value of the amplitude is expected to be stable in the infinitely long system, while the other pulse must be unstable, playing the role of a separatrix between attraction domains of the stable pulse and zero solution. These predictions have been completely confirmed by direct simulations. Moreover, if the integration domain is not very large (and periodic boundary conditions are imposed), some pulses are stable even when the zero background is unstable. An explanation to the latter feature, based on the concept of periodic suppression of the perturbations by the running pulse, is proposed. Furthermore, stable bound states of two and three identical pulses have been found numerically. An interesting issue, which is left for further work, is a possibility of formation of stable periodic arrays of the pulses. Note that periodic pulse arrays in the KS - KdV equation were studied in Refs. 88, 9.

Lastly, it is worthy to note that, since we have found stable pulses in the model including the dispersion term, i.e., $u_{x x x}$ in Eq. (3), a natural question is 
if the presence of this term is a necessary condition for the existence of stable pulses. Our preliminary numerical study demonstrates that there is a finite critical minimum value of the coefficient in front of the dispersive term (if all the other parameters of the system are fixed) which is indeed necessary for the existence of stable pulses. For instance, in a typical case considered in this work, with $c=0, \alpha=0.1, \gamma=0.05$, and $\Gamma=0.15$, this minimum value was found to be roughly 0.5 .

A plausible qualitative explanation for this effect (which will be considered in detail elsewhere) is that, although the dispersion does not directly affect the saturation mechanism stabilizing the pulses, it can inhibit mode coupling as an effective impedance, which will lead to attaining the necessary saturation at a higher amplitude of the pulse. Therefore, the amplitude of pulses becomes larger as the dispersion increases, while the pulse widths, directly determined by the dissipation, remain almost constant. This trend to the formation of pulses with higher amplitudes in the presence of strong dispersion is a feasible cause for the existence of stable pulses.

\section{Acknowledgments}

This work was performed, in a part, within the framework of an exchange program jointly supported by the Japan Society for Promotion of Science and Israeli Ministry of Science and Technology. B.A.M. appreciates hospitality of the Department of Aeronautics and Astronautics at the Kyoto University. The authors appreciate a valuable discussion with G.I. Sivashinsky.

\section{Appendix}

Here we give a detailed description of the numerical method employed for the simulation of Eqs. (5) and (6). First, we introduce necessary notation for the discrete Fourier transform (DFT). Suppose we work on the interval $I=[0, L]$ with $L$-periodic functions. The interval is discretized by means of a set of $N$ equidistant points, $x_{j}=j L / N$, where $j=0,1, \cdots, N-1$, with the spacing between them $\Delta x=L / N$. At these points, the numerical solutions for $u\left(x_{j}, t\right)$, $v\left(x_{j}, t\right)$ are denoted by $u_{j}(t), v_{j}(t)$ respectively. The corresponding spectral variable is $\xi_{k}=2 \pi k / L$ with $k \in\{-N / 2, \cdots,-1,0,1, \cdots, N / 2-1\}$ (actually, $N / 2$ is an integer, see below). Then DFT is given by

$$
\hat{u}_{k}=\mathcal{F} u_{j}=\sum_{j=0}^{N-1} u_{j} \exp \left(-i \xi_{k} x_{j}\right), \quad k=-\frac{N}{2}, \cdots,-1,0,1, \cdots, \frac{N}{2}-1 .
$$

The inverse DFT is defined as

$$
u_{j}=\mathcal{F}^{-1} \hat{u}_{k}=\frac{1}{N} \sum_{k=-N / 2}^{N / 2-1} \hat{u}_{k} \exp \left(i \xi_{k} x_{j}\right), \quad j=0,1, \cdots, N-1 .
$$


We employ the fast Fourier transform (FFT) to carry out the DFT and its inverse in the numerical form, therefore $N$ must be taken equal to a powers of 2.

The Fourier transform converts Eqs. (5), (6) into

$$
\begin{aligned}
\hat{u}_{t}+i \xi_{k} \mathcal{F}\left(\frac{1}{2} u^{2}\right)-i \xi_{k}^{3} \hat{u}-i \xi_{k} \hat{v} & =\alpha \xi_{k}^{2} \hat{u}-\gamma \xi_{k}^{4} \hat{u} \\
\hat{v}_{t}+i c \xi_{k} \hat{v}-i \xi_{k} \hat{u} & =-\Gamma \xi_{k}^{2} \hat{v}
\end{aligned}
$$

where $k=-N / 2, \cdots,-1,0,1, \cdots, N / 2-1$.

For the time integration of Eqs. 25) and (26), the Crank-Nicolson scheme is used, which leads to a nonlinear system

$$
\begin{aligned}
& \frac{\hat{u}^{n+1}-\hat{u}^{n}}{\Delta t}+\frac{1}{2} i \xi_{k}-i \xi_{k}^{3} \frac{\hat{u}^{n+1}+\hat{u}^{n}}{2}-i \xi_{k} \frac{\hat{v}^{n+1}+\hat{v}^{n}}{2} \\
& +\left(\mathcal{F}\left(\frac{\left(u^{n}\right)^{2}}{2}\right)+\mathcal{F}\left(\frac{\left(u^{n+1}\right)^{2}}{2}\right)\right)=\left(\alpha \xi_{k}^{2}-\gamma \xi_{k}^{4}\right) \frac{\hat{u}^{n+1}+\hat{u}^{n}}{2} \\
& \frac{\hat{v}^{n+1}-\hat{v}^{n}}{\Delta t}+i c \xi_{k} \frac{\hat{v}^{n+1}+\hat{v}^{n}}{2}-i \xi_{k} \frac{\hat{u}^{n+1}+\hat{u}^{n}}{2} \\
& =-\Gamma \xi_{k}^{2} \frac{\hat{v}^{n+1}+\hat{v}^{n}}{2} .
\end{aligned}
$$

To solve this nonlinear system, the following iteration procedure is employed:

$$
\begin{gathered}
\hat{u}^{n+1,0}=\hat{u}^{n}, \hat{v}^{n+1,0}=\hat{v}^{n} \\
\frac{\hat{u}^{n+1, r+1}-\hat{u}^{n}}{\Delta t}-i \xi_{k}^{3} \frac{\hat{u}^{n+1, r+1}+\hat{u}^{n}}{2}-i \xi_{k} \frac{\hat{v}^{n+1, r+1}+\hat{v}^{n}}{2} \\
+\frac{1}{2} i \xi_{k}\left(\mathcal{F}\left(\frac{\left(u^{n}\right)^{2}}{2}\right)+\mathcal{F}\left(\frac{\left(u^{n+1, r}\right)^{2}}{2}\right)\right)=\left(\alpha \xi_{k}^{2}-\gamma \xi_{k}^{4}\right) \frac{\hat{u}^{n+1, r+1}+\hat{u}^{n}}{2}, \\
\frac{\hat{v}^{n+1, r+1}-\hat{v}^{n}}{\Delta t}+i c \xi_{k} \frac{\hat{v}^{n+1, r+1}+\hat{v}^{n}}{2}-i \xi_{k} \frac{\hat{u}^{n+1, r+1}+\hat{u}^{n}}{2} \\
=-\Gamma \xi_{k}^{2} \frac{\hat{v}^{n+1, r+1}+\hat{v}^{n}}{2} .
\end{gathered}
$$

Here $r=0,1, \cdots, R-1$, where $R$ is the iteration number in each time step. In practice, the number of the Fourier modes was taken to be 256 or 512 , the typical time step was 0.01 and 0.02 , and the iteration was run twice in each time step. 


\section{References}

[1] T. Yamada and Y. Kuramoto, Progr. Theor. Phys. 56, 681 (1976).

[2] G.I. Sivashinsky, Acta Astronautica 4, 1177 (1977); Ann. Rev. Fluid Mech. 15, 179 (1983).

[3] A.A. Nepomnyashchy, Mekhanika Zhidk. Gaza (Izv. AN SSSR), No. 3, p. 28 (1974) [in Russian].

[4] G.Z. Gershuni, E.M. Zhukhovitsky, and A.A. Nepomnyashchy. Stability of Convective Flows (Nauka publishers: Moscow, 1989 [in Russian]).

[5] K. Kassler, A.K. Hobbs, and P. Metzener, Physica D 93, 23 (1996).

[6] A.P. Ivansky, Prikl. Mekh. Tekhn. Fiz., No. 2, p. 52 (1980) [in Russian].

[7] T. Kawahara and S. Toh, Phys. Fluids 28, 1636 (1985); also in Dynamical Problems in Soliton Systems, p. 153, ed. by S. Takeno (Springer: Berlin, 1985); Pure Appl. Math. 43, 95 (1989).

[8] S. Toh and T. Kawahara, J. Phys. Soc. Jpn. 54, 1257 (1985).

[9] T. Kawahara and S. Toh, Phys. Fluids 31, 2103 (1988).

[10] H.-C. Chang, E.A. Demekhin, and D.I. Kopelevich, Phys. Rev. Lett. 75, 1747 (1995).

[11] H.-C. Chang, Phys. Fluids 29, 3142 (1986).

[12] J.A. Gear and R. Grimshaw, Stud. Appl. Math. 70, 235 (1984).

[13] B.A. Malomed and H.G. Winful, Phys. Rev. E 53, 5365 (1996); J. Atai and B.A. Malomed, Phys. Rev. E 54, 4371-4374 (1996).

[14] J. Atai and B.A. Malomed, Phys. Lett. A 246, No. 5, 412 (1998)

[15] H. Sakaguchi and B.A. Malomed, Physica D 147, 273 (2000).

[16] L. Kalaugher, Fibre Systems Europe 5, No. 3, p. 19 (2001).

[17] M. van Saarloos, E. de Wit, and W. van Saarloos, Phys. Rev. Lett. 75, 3830 (1995).

[18] J. Atai and B.A. Malomed, Phys. Lett. A 244, 551 (1998).

[19] B.-F. Feng and T. Kawahara, Physica D 139, 301 (2000). 


\section{Figure Captions}

Fig. 1. The stability region for solitary pulses in the parametric plane $(\alpha, \Gamma)$ of the system (5), (5) for $\gamma=0.05$ and $c=0$. The zero solution is stable to the left of the dashed curve, and Eq. (19) produces two physical solutions to the right of the continuous curve. The symbols $\mathbf{x}$ and $\mathbf{o}$ mark points at which direct simulations show, respectively, that the solitary pulse is unstable or stable.

Fig. 2. The expected stability region for the solitary pulses in the parametric plane $(\alpha, \Gamma)$ for $\gamma=0.05$ and $c=-1$. The continuous and dashed curves have the same meaning as in Fig. 1.

Fig. 3. A typical result of an inelastic collision between two stable solitons with different velocities, simulated within the framework of the zero-order conservative system (12) with $c=0$ : (a) $t=0$, (b) $t=0$, (c) $t=40$, (d) $t=40$, the initial velocities of the two solitons are $s_{1}=4.236$ and $s_{2}=2.414$.

Fig. 4. The expected nearly vanishing stability region for the solitary pulses in the parametric plane $(\alpha, \Gamma)$ for $\gamma=0.05$ and $c=0.3$. The continuous and dashed curves have the same meaning as in Fig. 1.

Fig. 5. Suppression of the initially imposed large perturbation which is the fastest growing instability mode in the infinite system by the traveling pulse in the case $\alpha=0.15, \Gamma=0.2, \gamma=0.05$, and $c=0$, in the spatial domain of the length $L=128$ with periodic boundary conditions. The perturbation is taken as $u_{\text {pert }}=v_{\text {pert }}=a_{0} \cos \left(k_{\max } x\right)$ with $a_{0}=1$ and $k_{\max }=1.3$. The panels (a) and (b) show the initial configurations of the fields, and (c) and (d) are their shapes produced by the direct simulations by the moment $t=420$.

Fig. 6. The instability of the pulse at the same values of parameters and for the same time interval as in Fig. 5, but in the spatial domain of the length four times as large, $L=512$, without any specially imposed initial perturbation.

Fig. 7. A stable bound state of two pulses found in the case $\alpha=0.1$, $\gamma=0.05, c=0, \Gamma=0.15$. The panels (a) and (b) show established shapes of the $u$ and $v$ fields.

Fig. 8. A stable bound state of three pulses in the same case as in Fig. 7. 


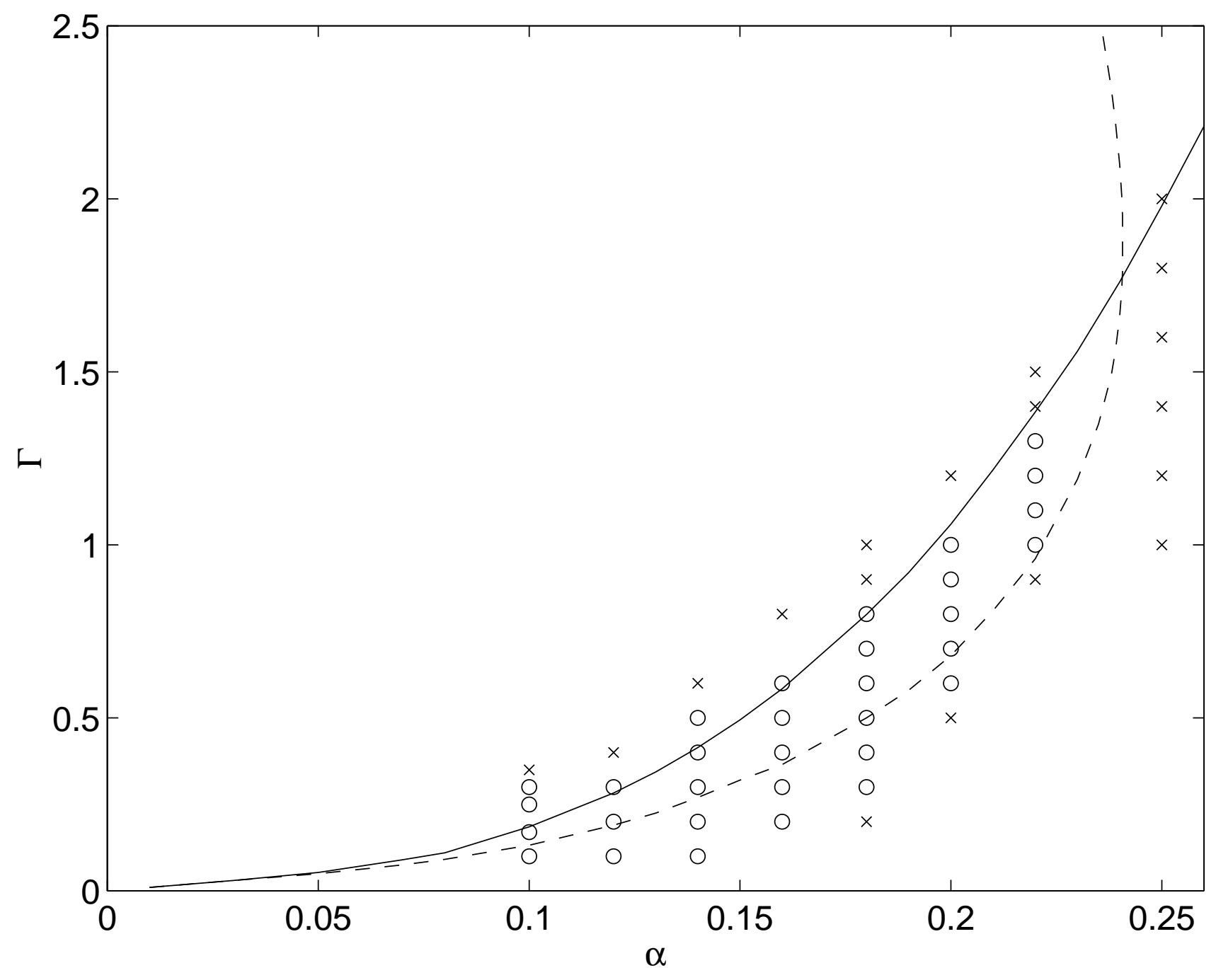




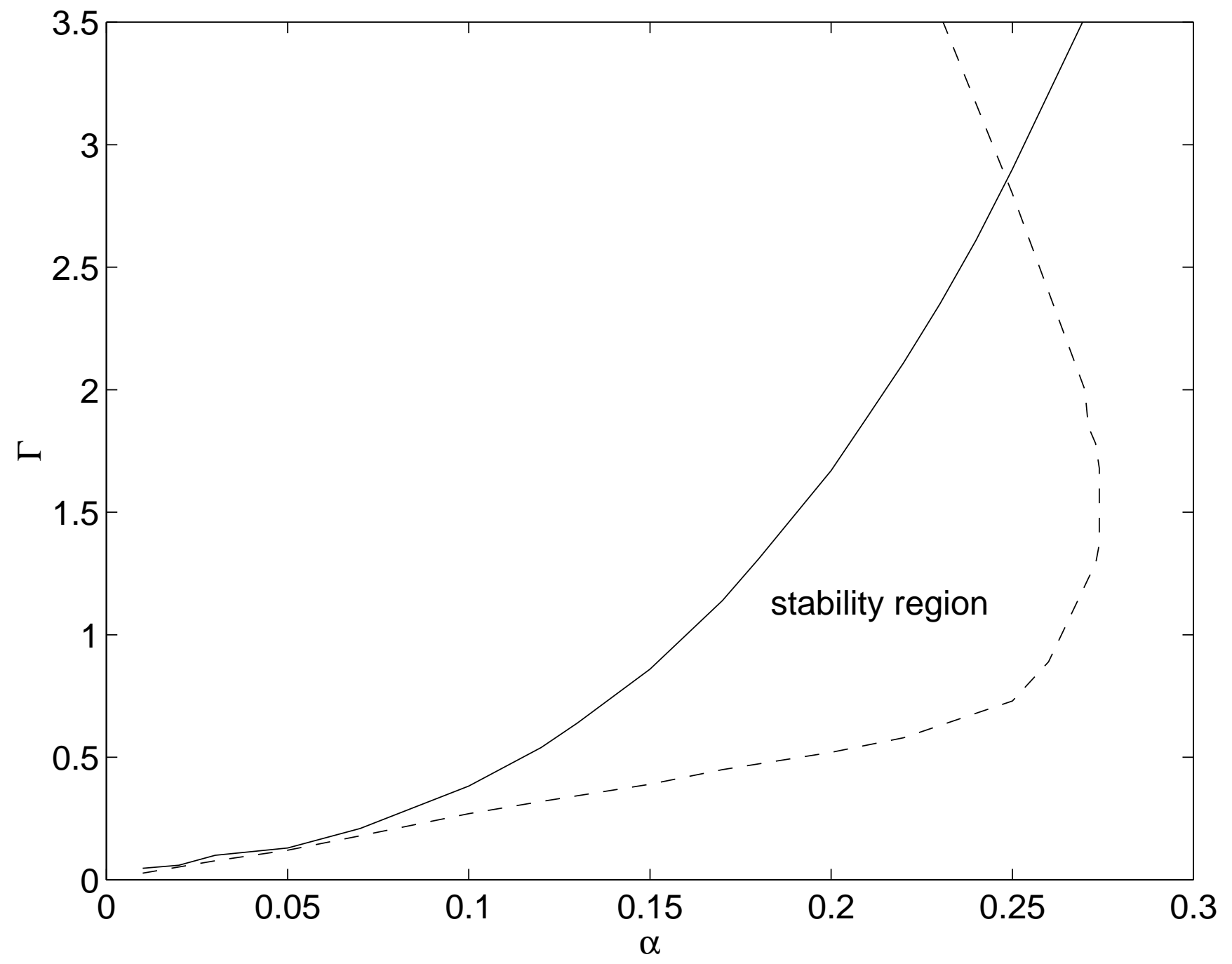




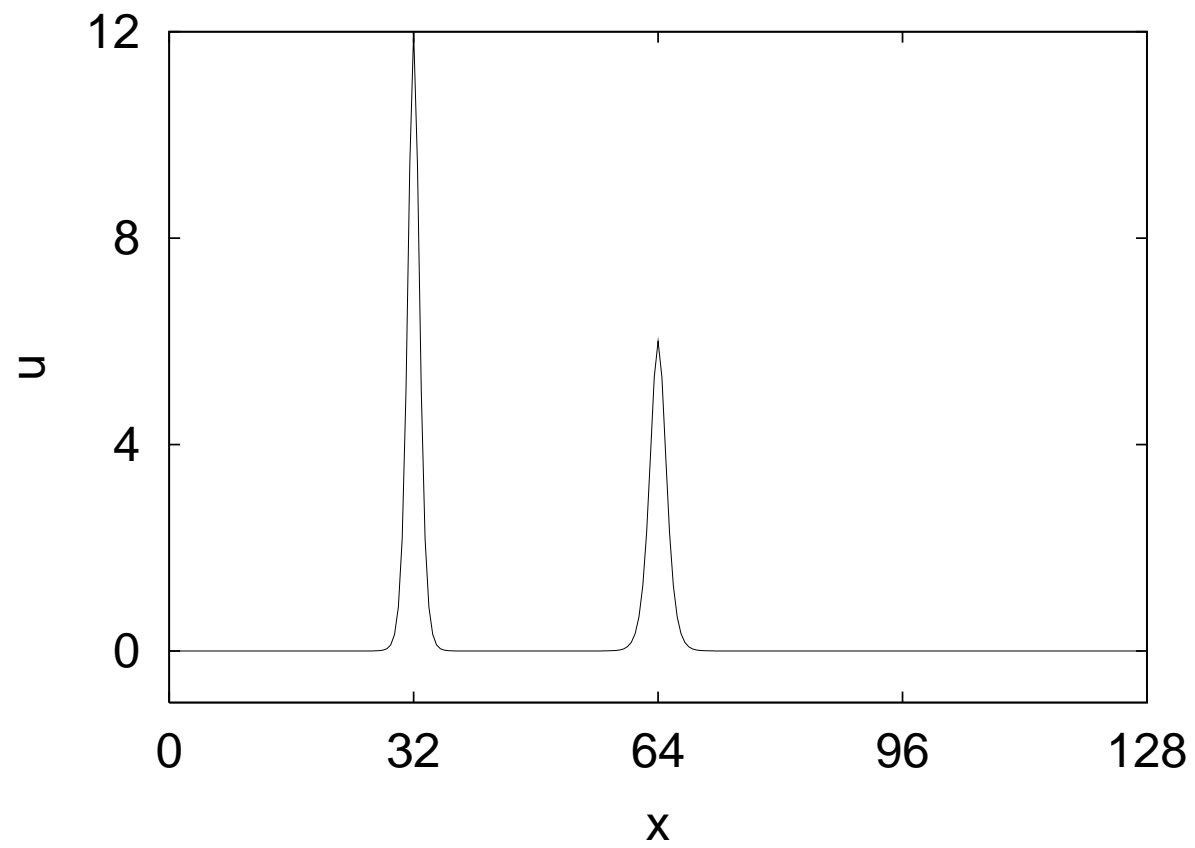




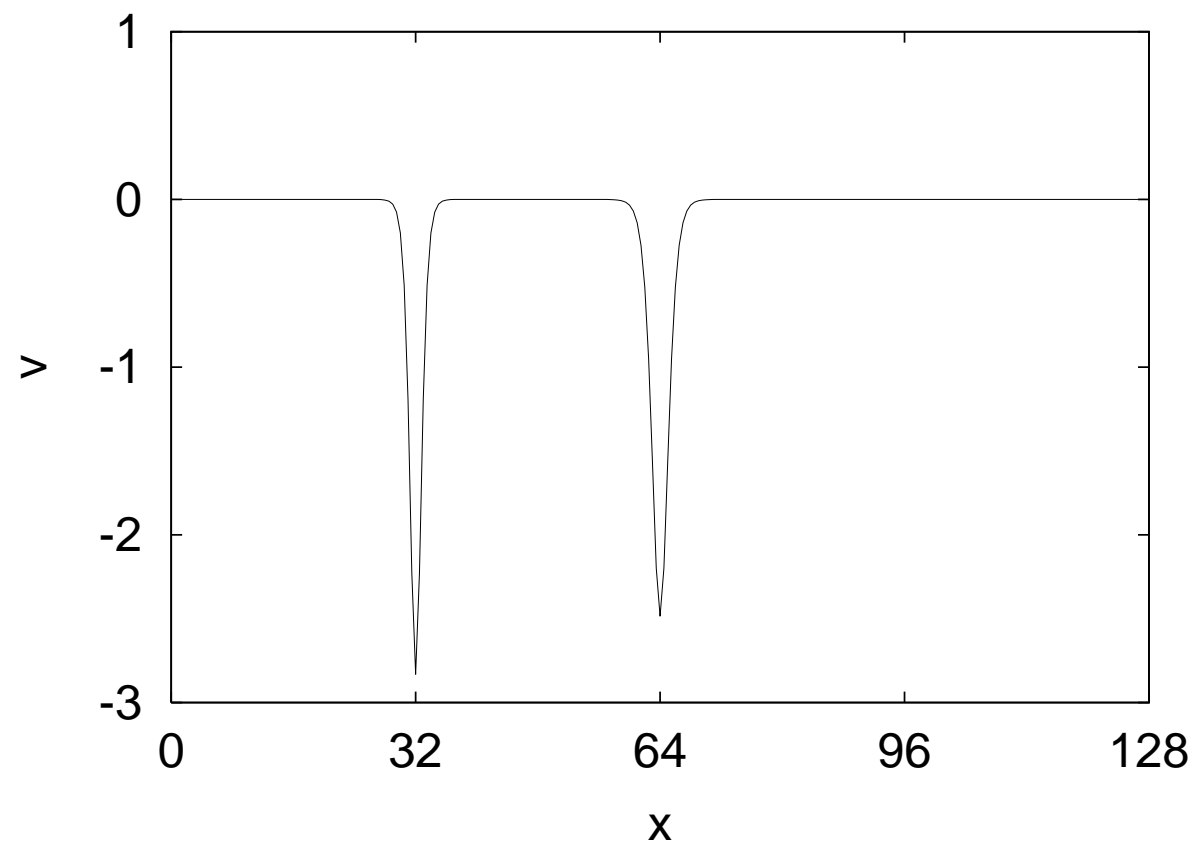




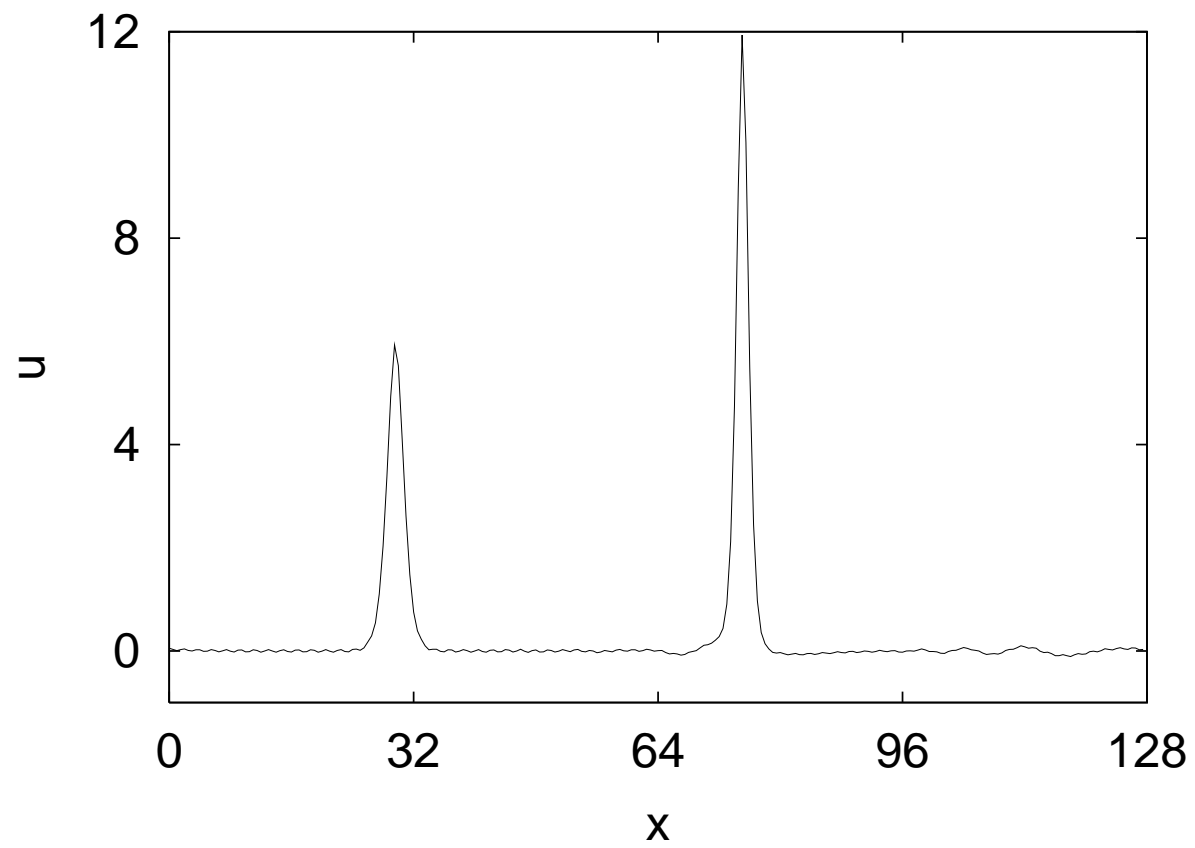




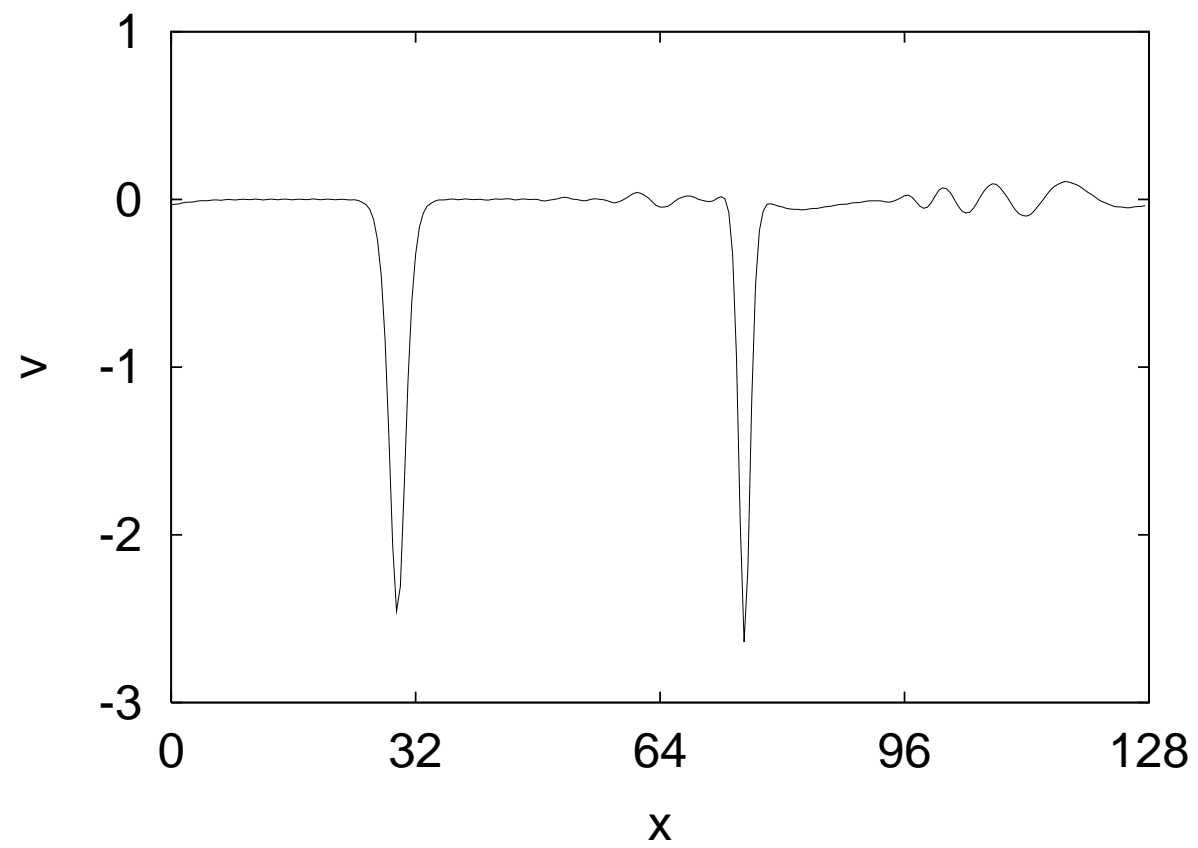




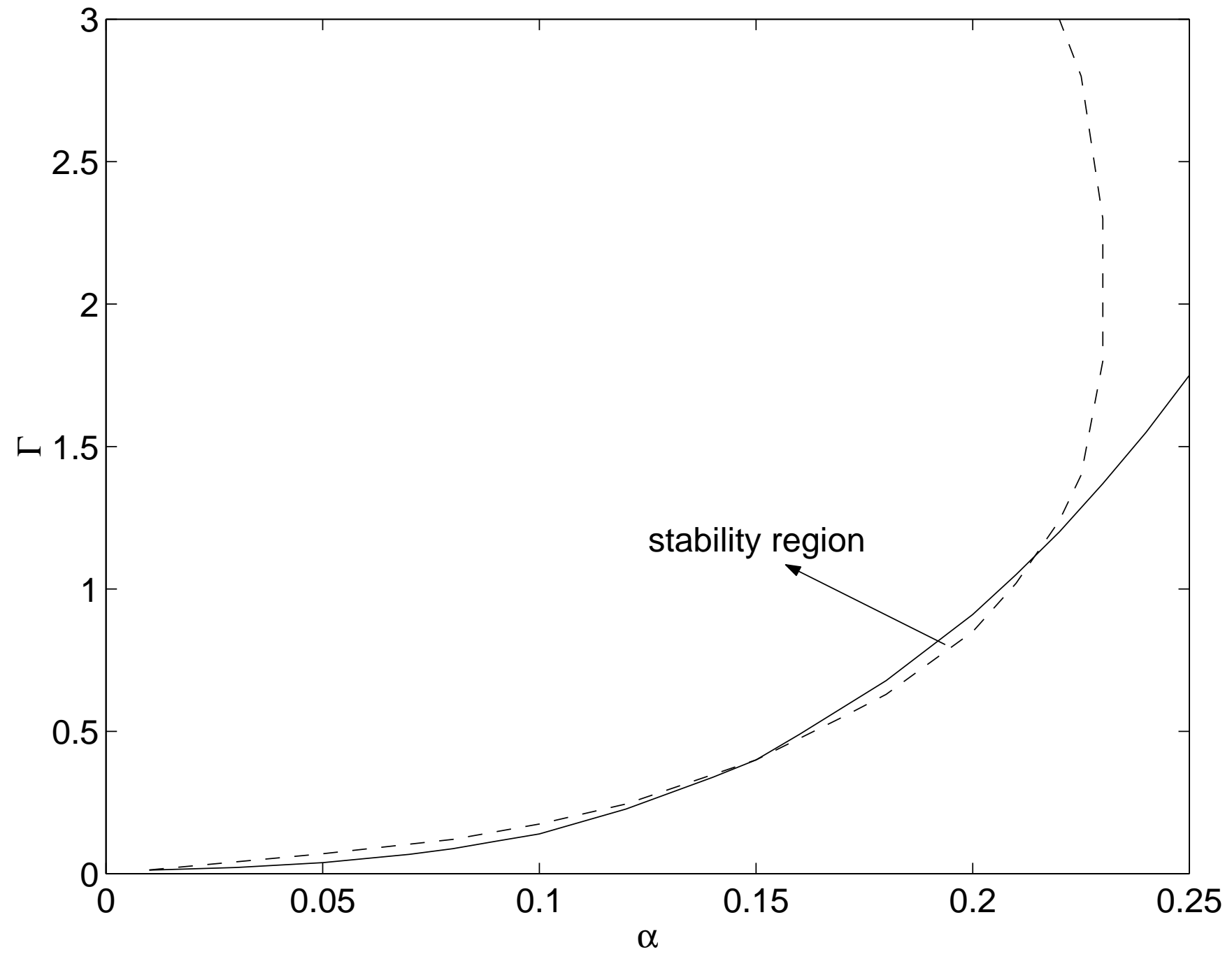




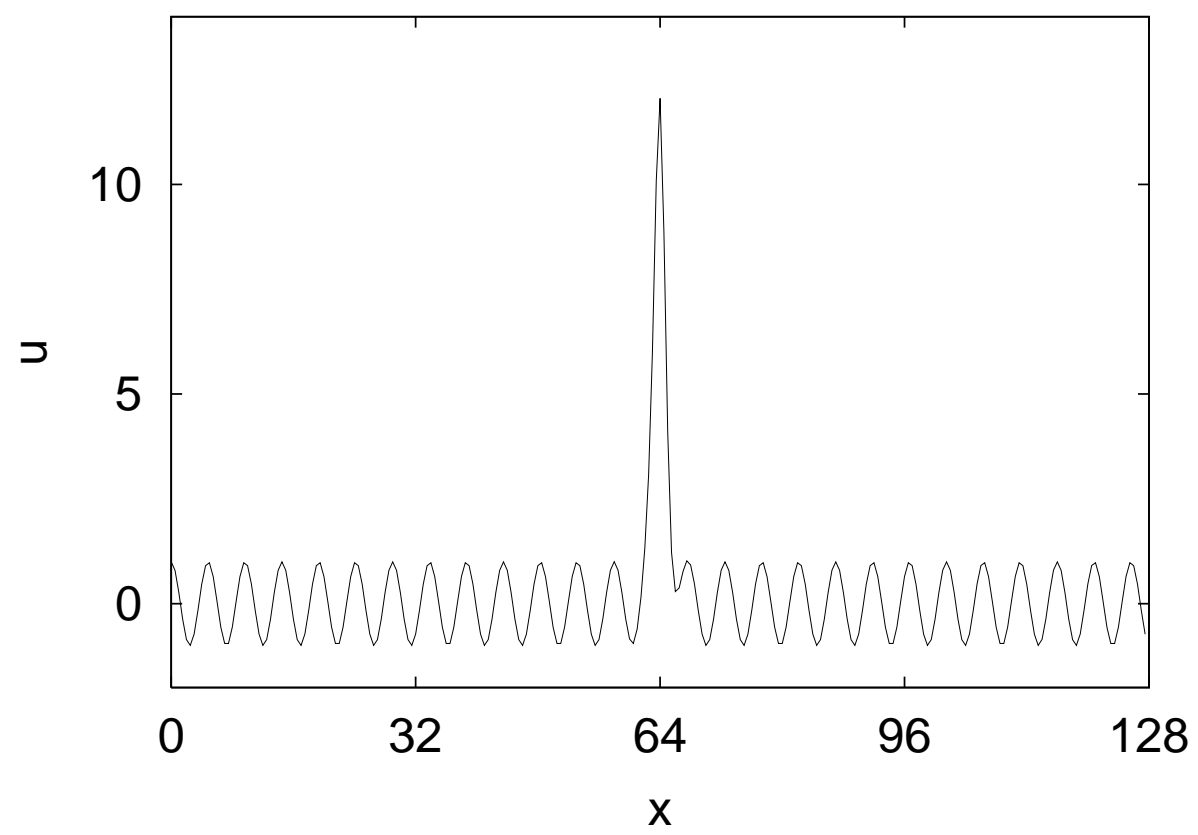




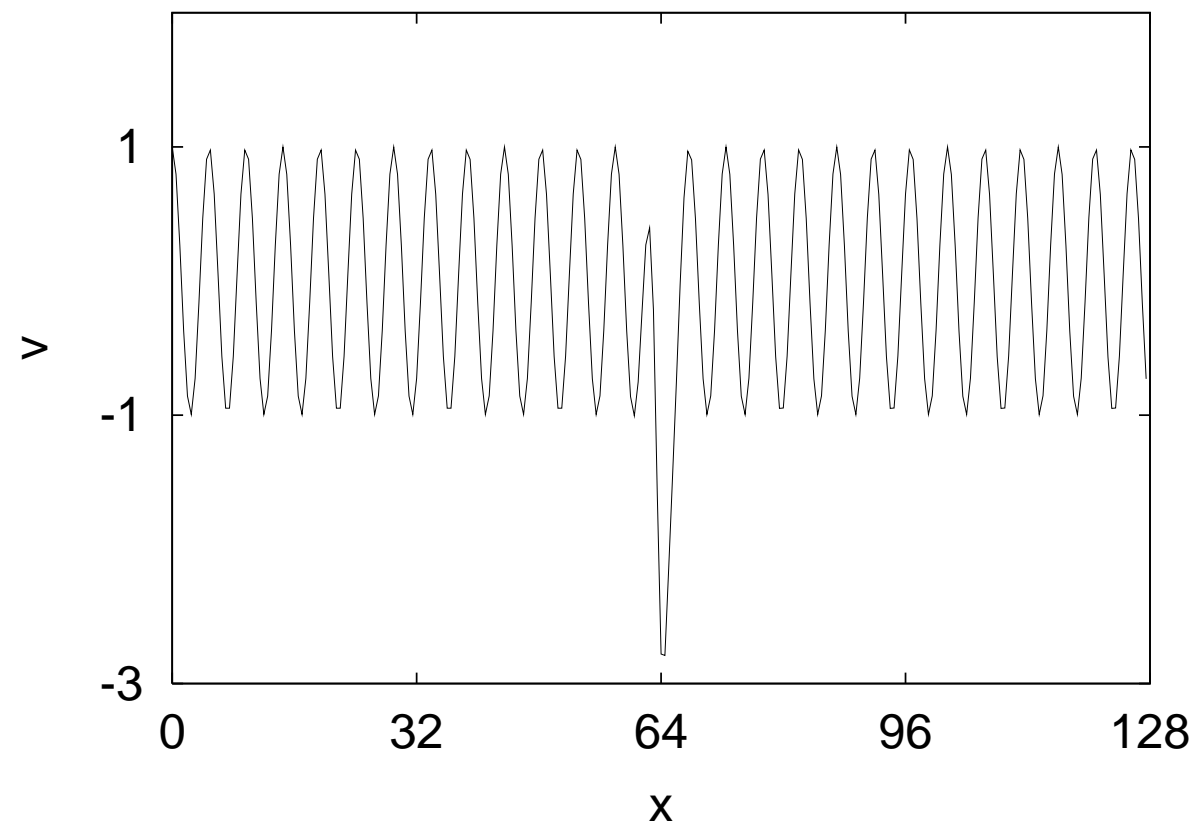




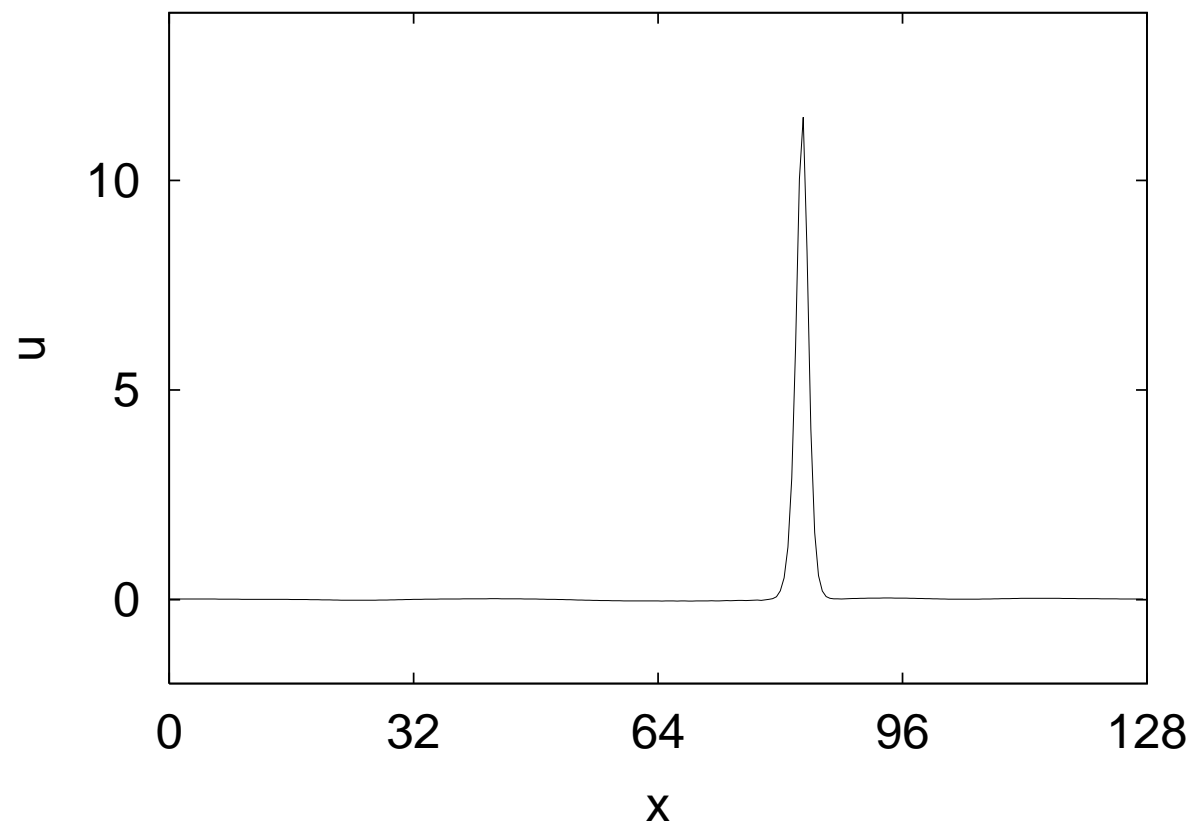




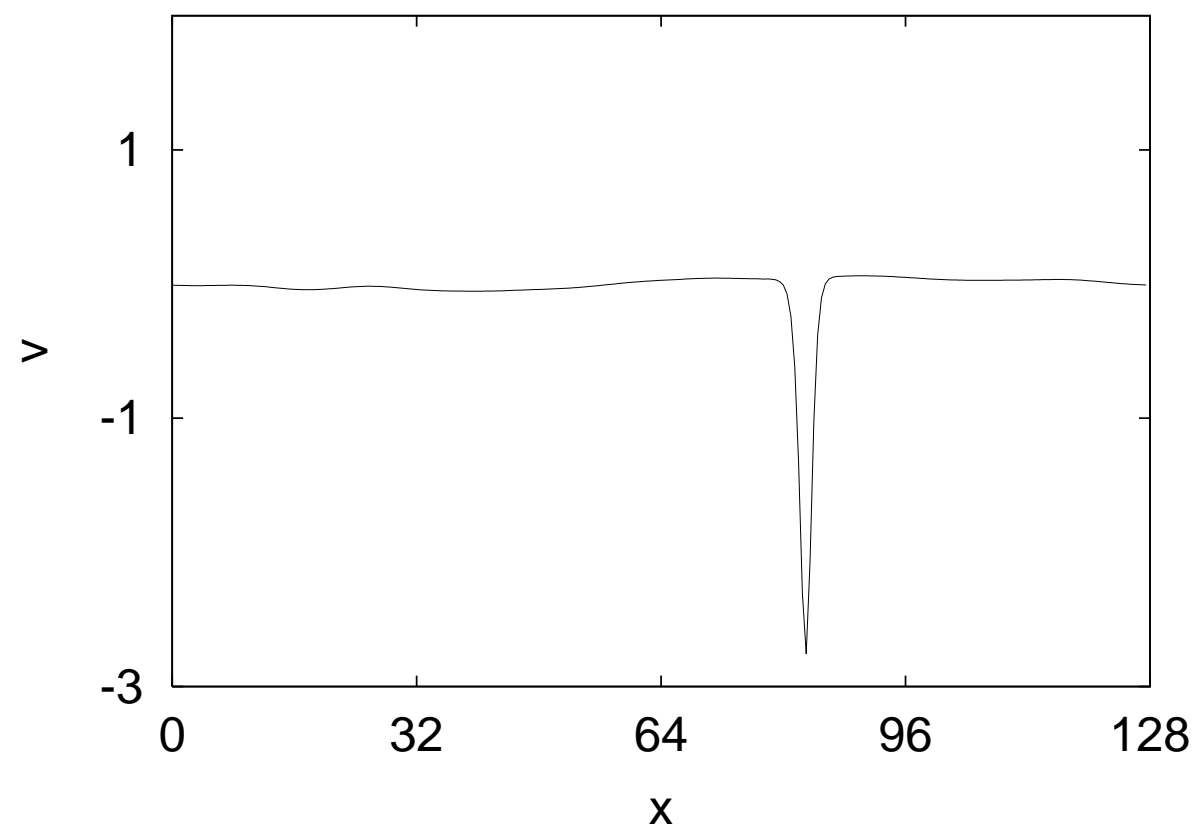




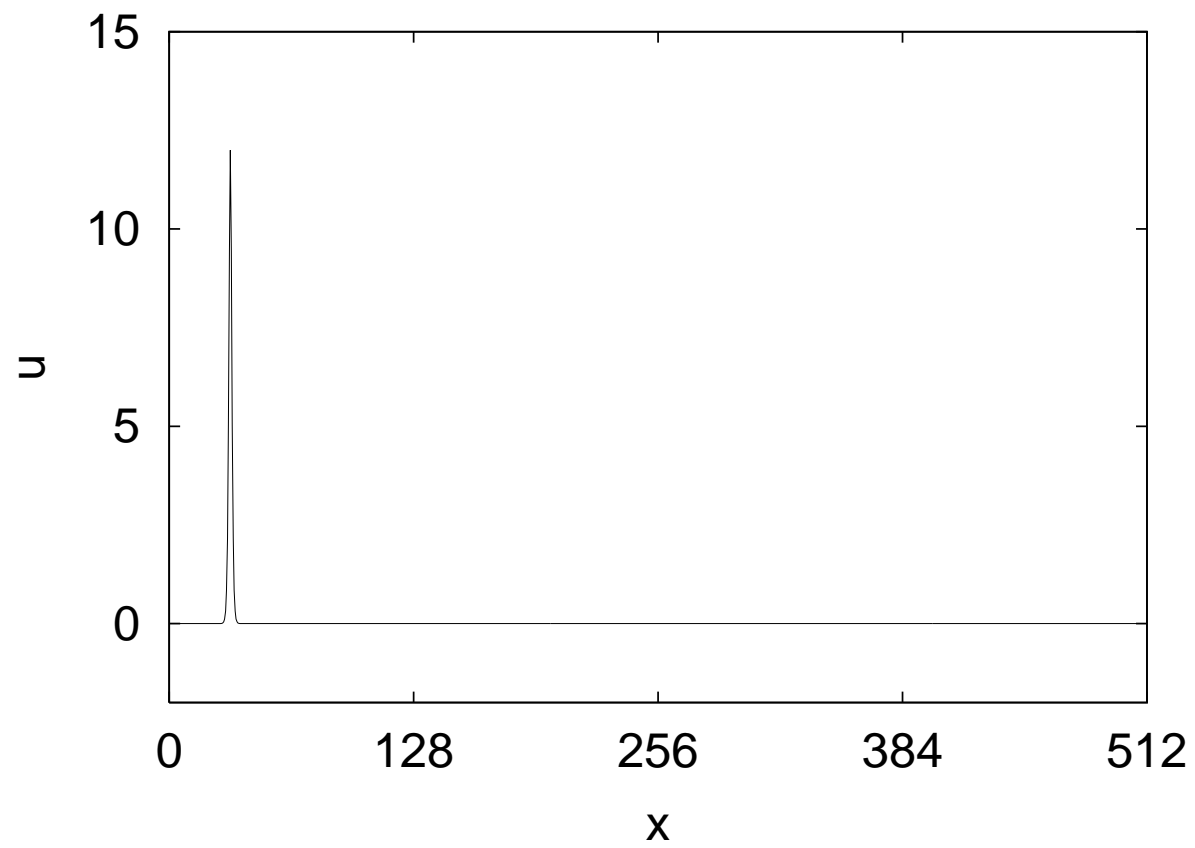




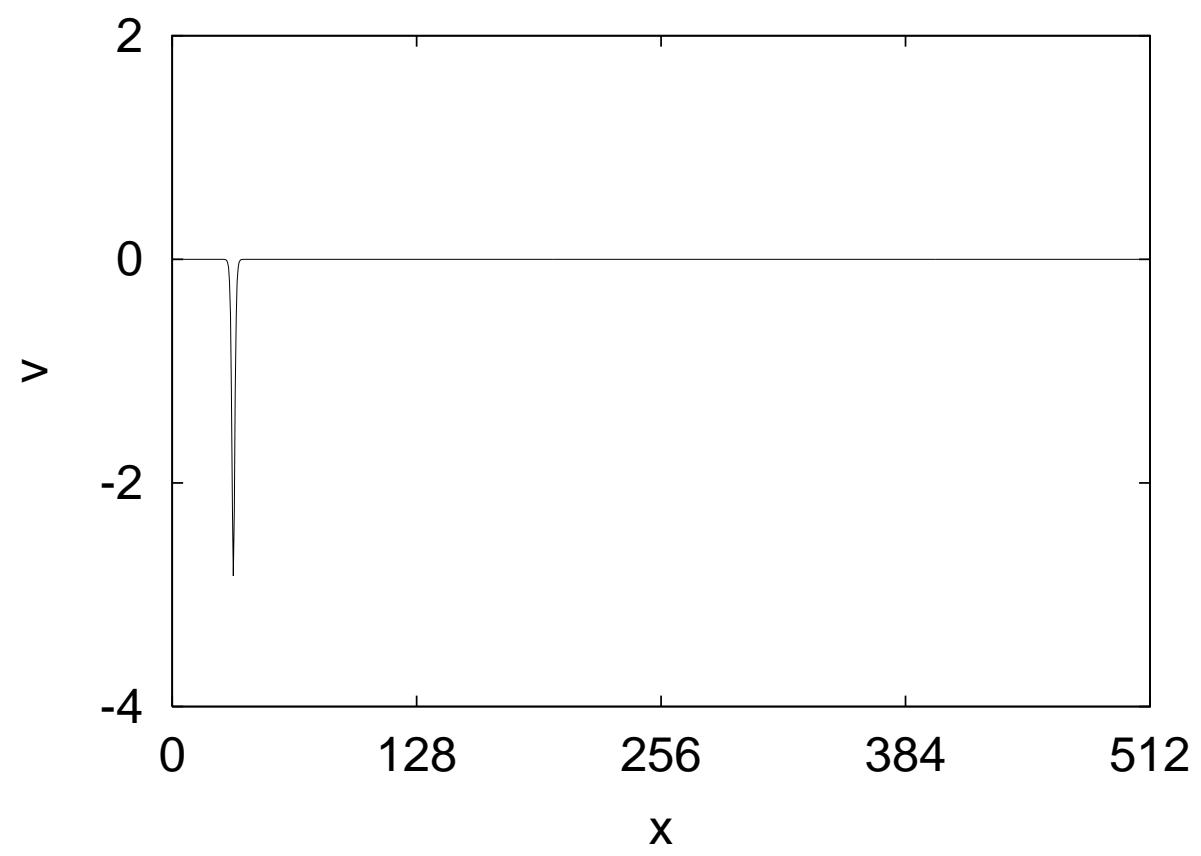




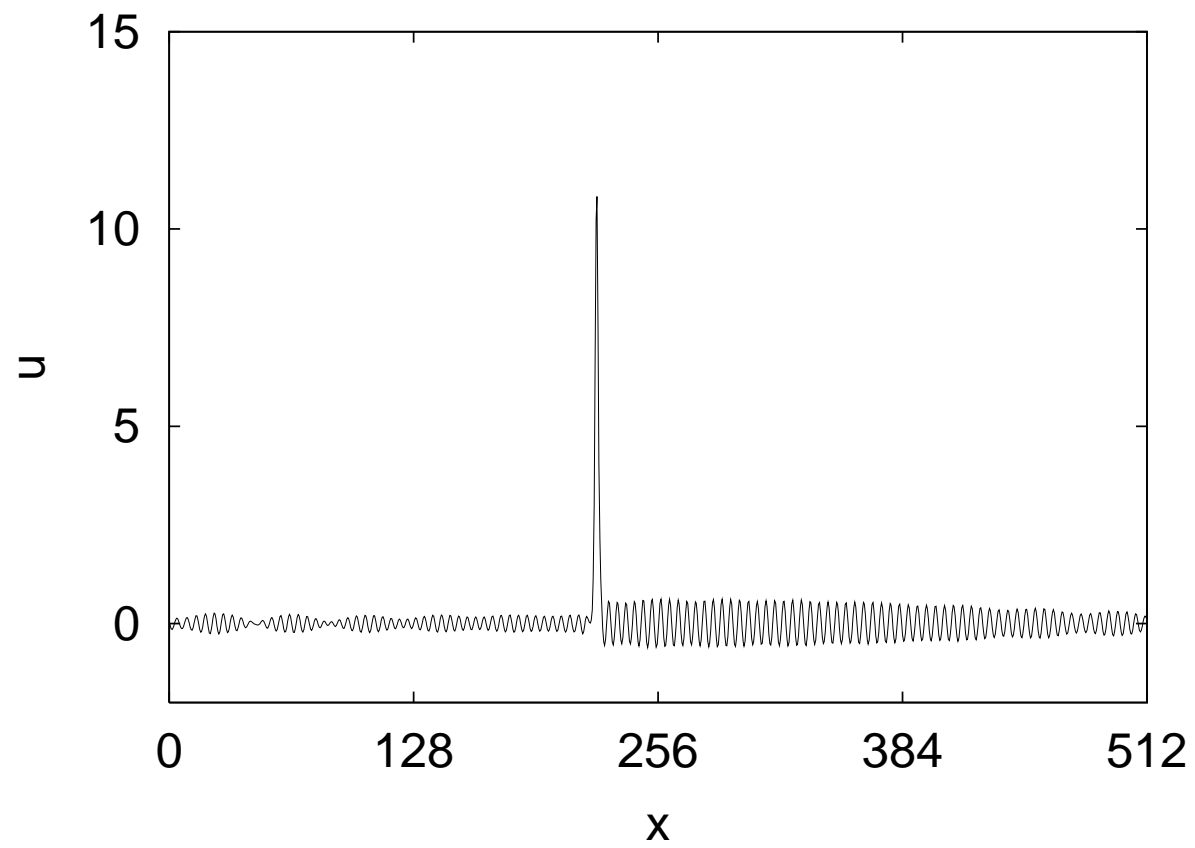




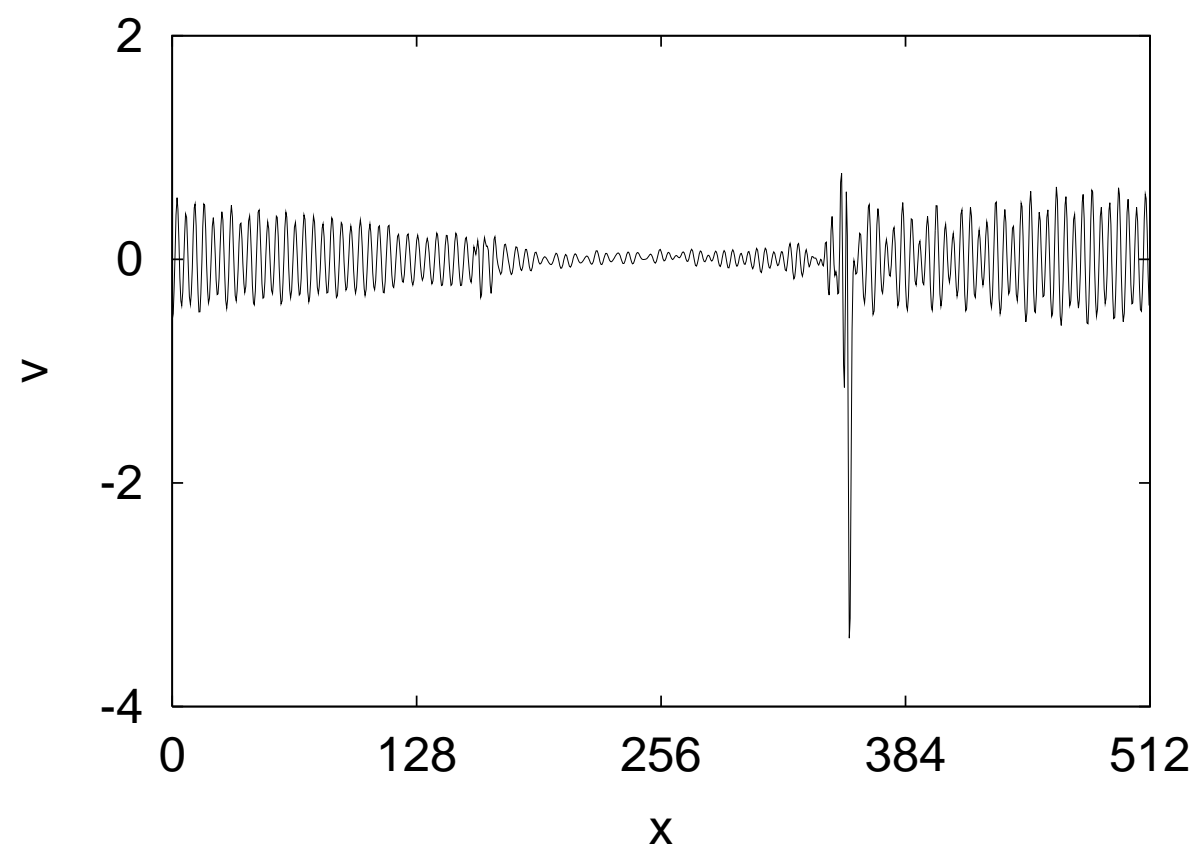




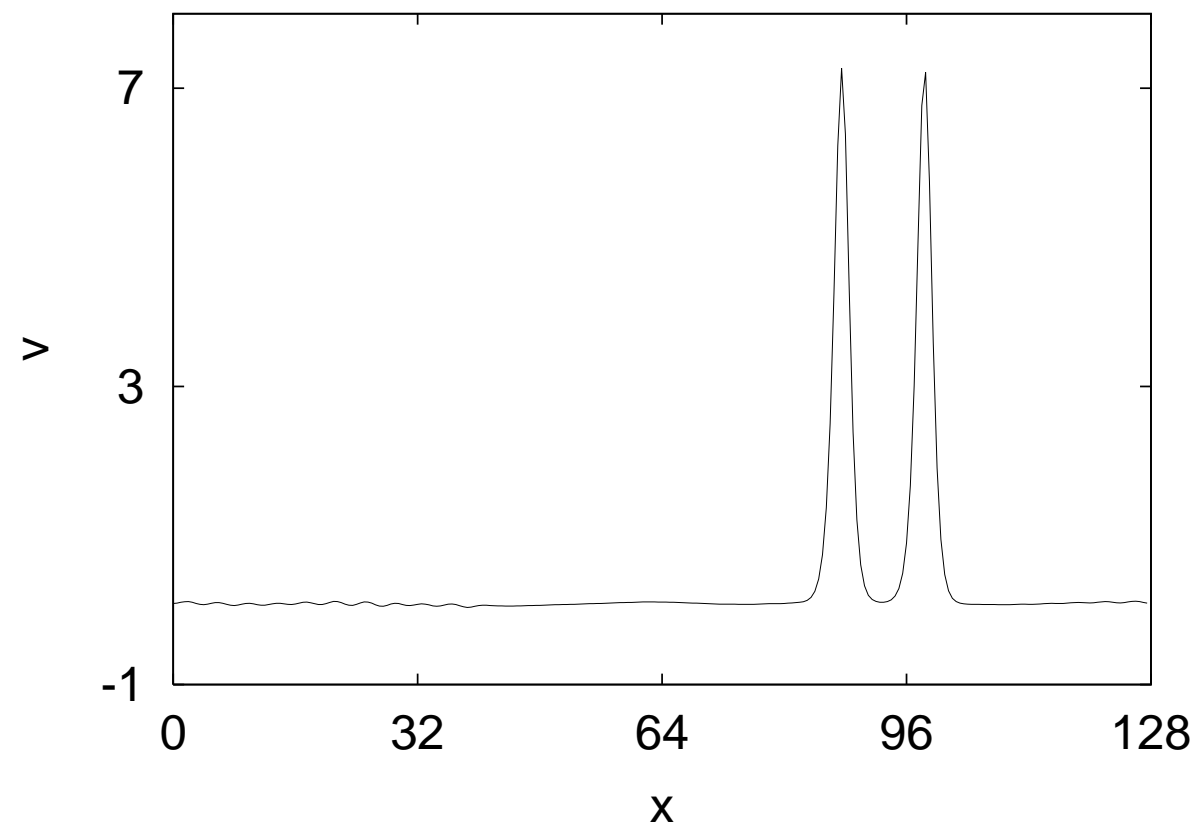




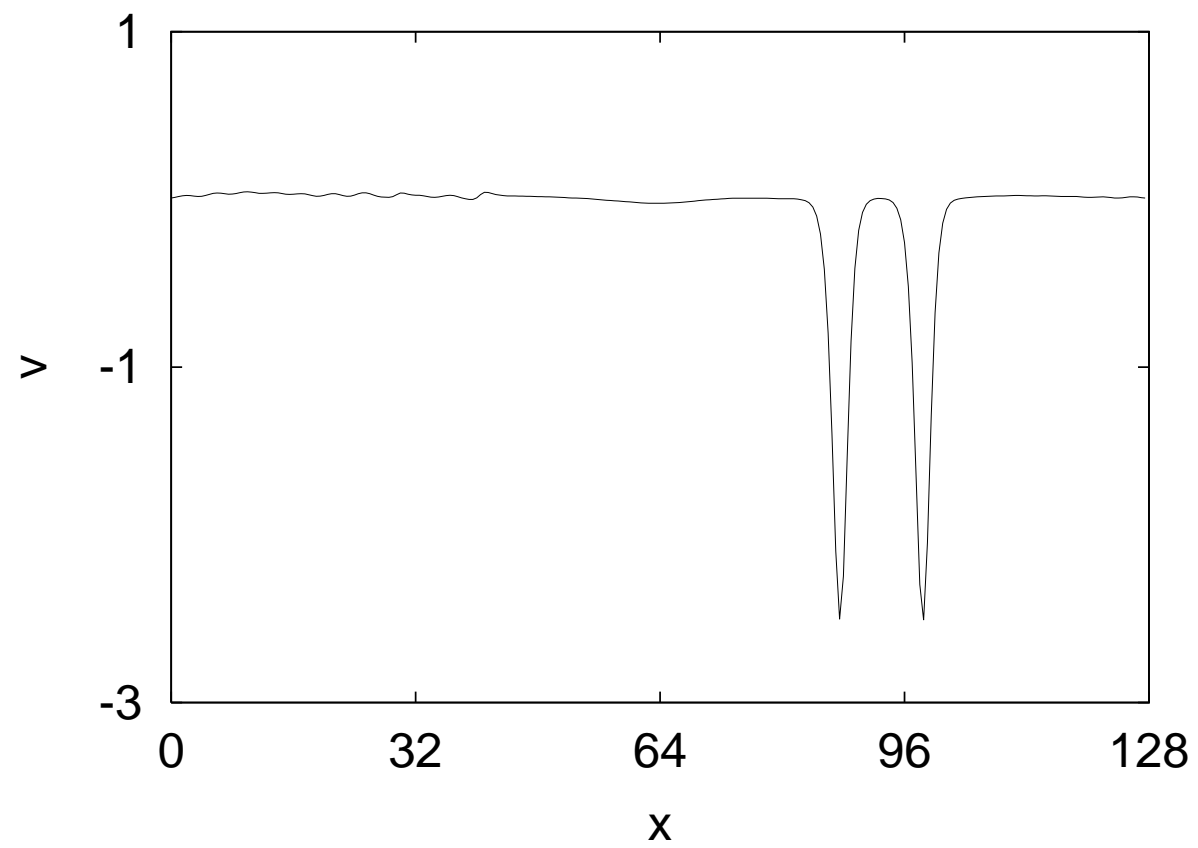




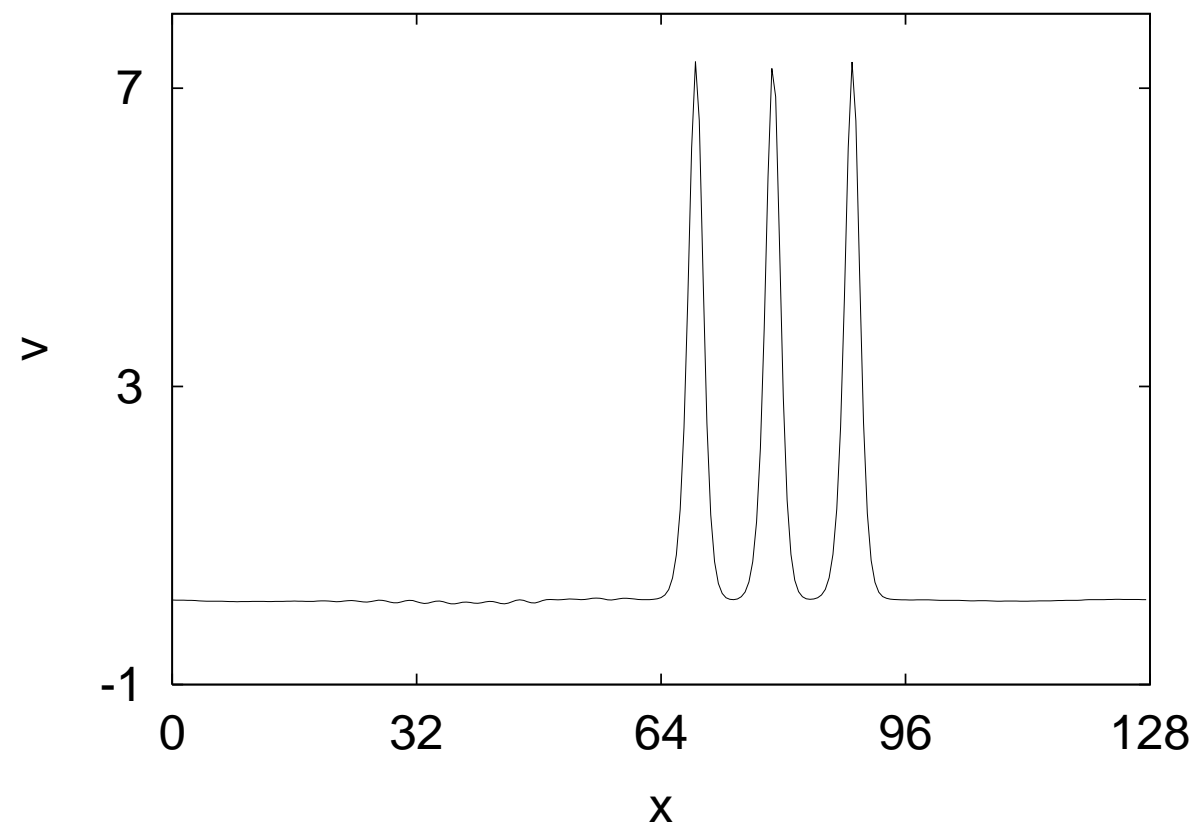




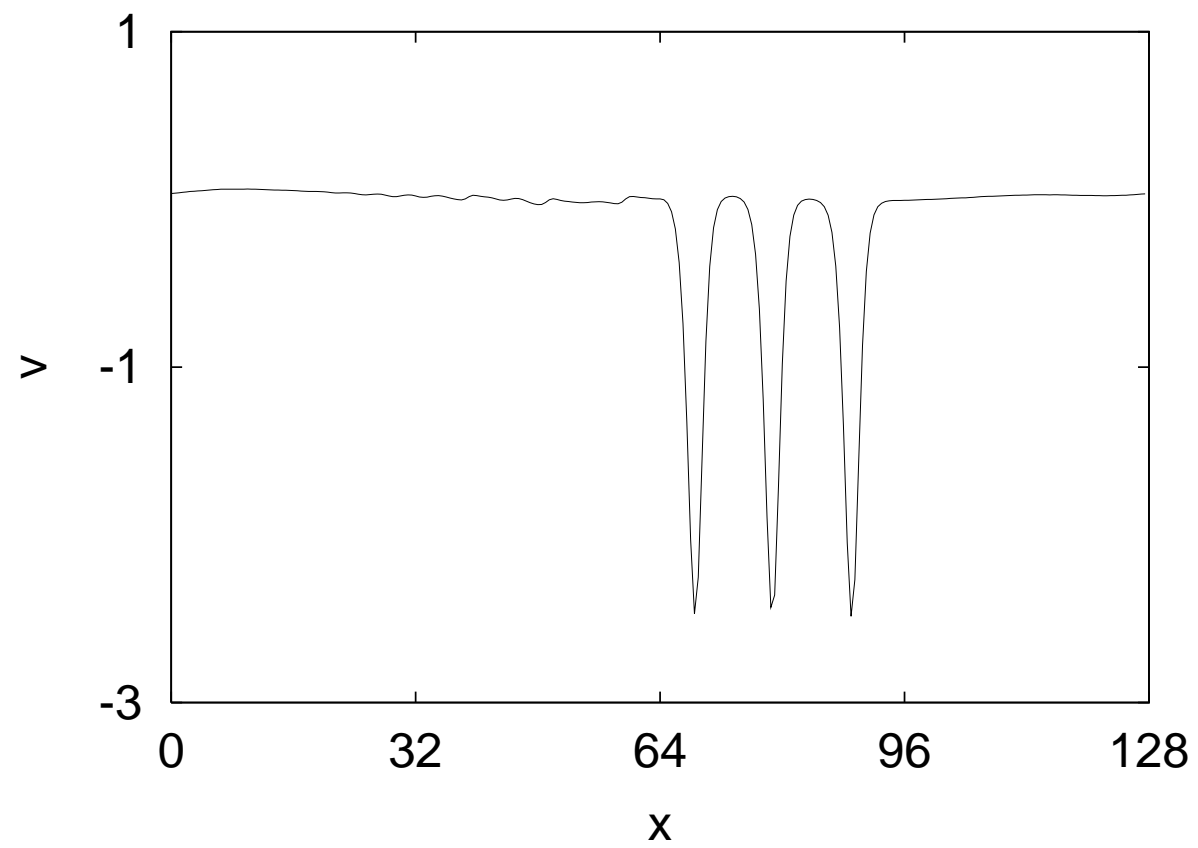

